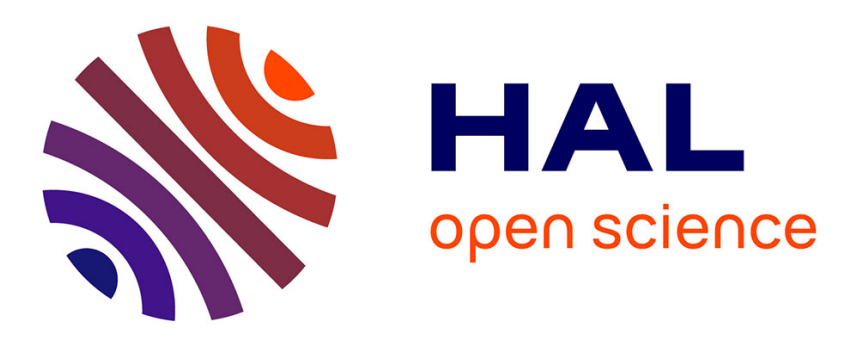

\title{
Supercontinuum generation using continuous-wave multiwavelength pumping and dispersion management
}

Thibaut Sylvestre, Armand Vedadi, Hervé Maillotte, Frédérique Vanholsbeeck, Stéphane Coen

\section{- To cite this version:}

Thibaut Sylvestre, Armand Vedadi, Hervé Maillotte, Frédérique Vanholsbeeck, Stéphane Coen. Supercontinuum generation using continuous-wave multiwavelength pumping and dispersion management. Optics Letters, 2006, 31 (13), pp.2036-2038. 10.1364/OL.31.002036 hal-00269935

\section{HAL Id: hal-00269935 \\ https://hal.science/hal-00269935}

Submitted on 29 Aug 2013

HAL is a multi-disciplinary open access archive for the deposit and dissemination of scientific research documents, whether they are published or not. The documents may come from teaching and research institutions in France or abroad, or from public or private research centers.
L'archive ouverte pluridisciplinaire HAL, est destinée au dépôt et à la diffusion de documents scientifiques de niveau recherche, publiés ou non, émanant des établissements d'enseignement et de recherche français ou étrangers, des laboratoires publics ou privés. 


\title{
Supercontinuum generation using continuous-wave multiwavelength pumping and dispersion management
}

\author{
Thibaut Sylvestre, Armand Vedadi, and Hervé Maillotte \\ Département d'Optique P. M. Duffieux, Institut FEMTO-ST, Université de Franche-Comté, Centre National \\ de la Recherche Scientifique, Unit Mixte de Recherche 6174, F-25030 Besançon, France \\ Frédérique Vanholsbeeck and Stéphane Coen \\ Department of Physics, The University of Auckland, Private Bag 92019, Auckland, New Zealand
}

\begin{abstract}
Received February 15, 2006; revised March 27, 2006; accepted March 27, 2006; posted April 6, 2006 (Doc. ID 68129) We experimentally demonstrate that continuous-wave supercontinuum generation in optical fibers can be significantly enhanced by using both multiwavelength pumping and dispersion management. We show by detailed spectral analysis that continuum enhancement is achieved mainly through a combination of Raman-assisted modulation instabilities, soliton compression, and dispersive wave generation. With this technique, an $800 \mathrm{~nm}$ wide (from 1.2 to $2.0 \mu \mathrm{m}$ ) $2 \mathrm{~W}$ supercontinuum source is reported that uses a threewavelength pump and a dispersion-tailored four-optical fibers arrangement. (c) 2006 Optical Society of America

OCIS codes: $060.4370,060.7140,190.5530$.
\end{abstract}

Supercontinuum (SC) generation has recently been achieved simply with high-power cw fiber lasers and standard dispersion-shifted optical fibers (DSFs). ${ }^{1-7}$ These new highly efficient, compact, all-fiber SC sources at selectable bands exhibit a high power spectral density (up to $10 \mathrm{~mW} / \mathrm{nm}$ ) as well as excellent power stability and spectral smoothness, usually better than pulsed SC sources. ${ }^{2,6}$ They appear promising as solutions for many advanced applications such as component testing, optical communications, metrology, and optical coherence tomography.

The general principle for SC generation in the $\mathrm{cw}$ regime is similar to that of the pulsed regime, except that the initial $\mathrm{cw}$ field is first converted into a train of ultrashort pulses through modulation instability (MI) in the low anomalous dispersion regime of DSFs. ${ }^{3,8}$ These solitonlike pulses then undergo fission due to stimulated Raman scattering (SRS) and third-order dispersion and shed radiation in the form of blueshifted dispersive waves (DWs). ${ }^{3,9}$ SRS also plays a major role in the cw regime in enhancing the spectral broadening towards longer wavelengths through the Raman self-frequency shift of ultrashort pulses. Finally, the partially coherent nature of the input field acts as a seed for the MI-induced SC generation and helps smooth the resulting spectrum. $3,6,10$ In this way, cw-SC can be efficiently generated in many spectral regions depending on the cw pump wavelength and on the fiber dispersion. ${ }^{1,3-6}$

With regard to practical applications, both the uniformity and the width of the SC spectrum are, however, important issues. In this respect, it is significant that single-pump cw-SC sources generally exhibit a limited bandwidth in comparison with what can be achieved in the pulsed regime. Recently it has been predicted theoretically that dispersion management can enhance cw-SC generation by increasing the soliton order of the generated pulses, which leads to stronger temporal compression and higher intensities. ${ }^{11}$ Experiments performed in photonic crystal fibers with picosecond pump pulses have also shown that dispersion management can enhance phase-matched four-wave mixing and extend the SC bandwidth. $^{12}$ Here we propose and experimentally demonstrate an extension of this technique by combining dispersion management with multiwavelength cw pumping. In particular, we report the generation of a broadband single-mode high-power $(2 \mathrm{~W})$ cw-SC that spans more than $800 \mathrm{~nm}$ (from 1.2 to $2.0 \mu \mathrm{m}$ ) based on an arrangement of three pump wavelengths and four optical fibers.

The experimental setup for SC generation is schematically depicted in Fig. 1. Two Raman fiber lasers (RFLs) are combined through a wavelength division multiplexer (WDM) into a hybrid fiber made up of one to four fiber spans with different lengths and dispersive and nonlinear properties. In the results presented below, we successively cascade a $3.1 \mathrm{~km}$ long conventional DSF with a zero-dispersion wavelength (ZDW) of $1549 \mathrm{~nm}$, two $500 \mathrm{~m}$ highly nonlinear DSFs (HNL-DSF, nonlinear coefficient $\gamma=11.8 \mathrm{~W}^{-1} \mathrm{~km}^{-1}$ ) with ZDWs at 1551 and $1553 \mathrm{~nm}$, respectively, and eventually a $2 \mathrm{~km}$ standard single-mode fiber (SMF) with a $1302 \mathrm{~nm}$ ZDW. The first laser (RFL1) is a dualorder $\mathrm{P}_{2} \mathrm{O}_{5}$-doped $\mathrm{RFL}$ delivering up to $2 \mathrm{~W}$ at a $1555 \mathrm{~nm}$ wavelength and a $0.7 \mathrm{~nm} 3 \mathrm{~dB}$ linewidth. It also emits a weaker first-order Raman component at $1289 \mathrm{~nm}$ ( $1.5 \mathrm{~nm}$ linewidth) that is used in the fourfiber arrangement. The second laser (RFL2) is a standard 5 W Raman laser, more powerful than RFL1,

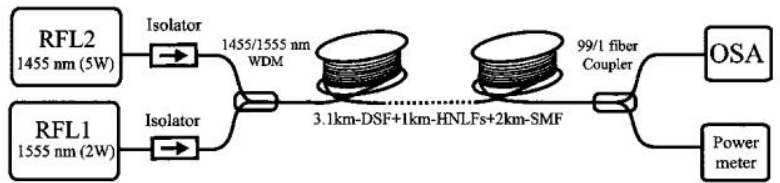

Fig. 1. Experimental setup. OSA, optical spectrum analyzer. 
with an output wavelength of $1455 \mathrm{~nm}$ and a $3 \mathrm{~dB}$ linewidth of $1.2 \mathrm{~nm}$.

We must point out that the wavelength spacing between the two lasers has been chosen to match exactly the peak of the Raman gain of silica-based fibers $\left(\Omega_{R}=13.2 \mathrm{THz}\right)$. Consequently, we can expect a strong distributed amplification of the $1555 \mathrm{~nm}$ line of RFL1 by the second laser and a corresponding enhancement of SC generation. To demonstrate this Raman-assisted SC generation process, we carried out a first set of cw-SC generation experiments using dual-wavelength pumping in only the first span of DSF. Figure 2 shows the experimental output SC spectra for a fixed RFL1 pump power at $1555 \mathrm{~nm}$ and an increasing RFL2 pump power. When the second pump is absent (second curve from bottom), the RFL1 pump is first spectrally broadened by MI because of the small anomalous dispersion. Moreover, blueshifted DWs emitted from soliton fission can be seen on the short-wavelength side of the spectrum. When RFL2 is turned on, enhanced SC generation can clearly be seen in the top three plots of Fig. 2, recorded for different RFL2 pump powers. New spectral components are generated both between the two pumps and on the long-wavelength side of RFL1, extending the spectrum beyond the $1.7 \mu \mathrm{m}$ limit of the optical spectrum analyzer (OSA). The level of the residual $1455 \mathrm{~nm}$ pump at the fiber output has been measured to be reduced by $40 \mathrm{~dB}$ when coupled with the RFL1 pump, revealing an excellent power conversion efficiency from the Raman pump RFL2 to the $\mathrm{SC}$, and this leads to more than a fourfold increase in the SC bandwidth. Physically, it is obvious that both new Raman solitons and new DWs with large frequency shifts are generated both on the long- and short-wavelength sides of the SC spectrum. Additionally, Fig. 2 reveals two small narrow lines at 1651 and $1670 \mathrm{~nm}$ (indicated by arrows). These lines are initially generated by four-wave mixing (FWM) between the Raman pump RFL2 and the continuum and subsequently experience Raman amplification and spectral broadening. In this way, these so-called

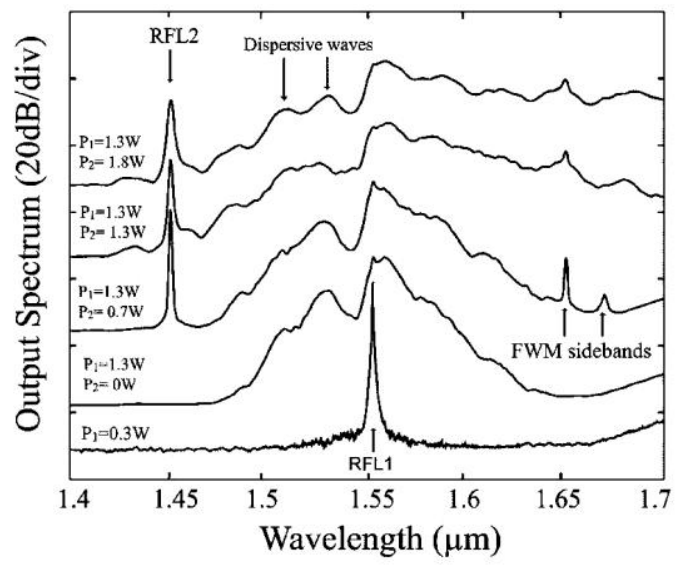

Fig. 2. Experimental output SC spectra generated in the first DSF span without (two bottom curves) and with dualwavelength pumping (three top curves). Pump power levels are indicated on the plot.

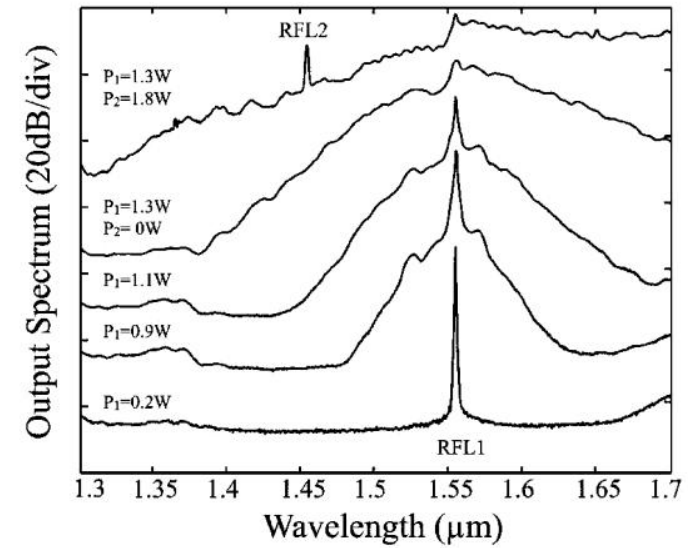

Fig. 3. Experimental output SC spectra generated in the two-fiber arrangement with a single RFL1 pump (four bottom curves) and in combination with dual-wavelength pumping (top curve).

Raman-assisted FWM processes ${ }^{13,14}$ improve the SC width and smoothness toward long wavelengths.

In the second part of our experimental analysis, we consider dispersion management for enhancing SC generation. To this end, we add one $500 \mathrm{~m}$ span of HNL-DSF behind the $3.1 \mathrm{~km}$ long DSF. The experimental output spectra are shown in Fig. 3 for increasing pump power (from bottom to top) when using only RFL1, and this reveals a much broader and smoother SC in comparison with the simple one-fiber arrangement. In our configuration, both fibers exhibit the same product $\gamma L$ of nonlinear coefficient times length. Under such conditions, we can already expect a significant broadening of the SC spectrum simply because of the additional length of nonlinear fiber. On top of this, the second fiber section has a lower second-order dispersion coefficient $\left(\beta_{2}\right)$. The short Raman solitons generated in the first fiber span will therefore undergo further temporal compression, intensity increase, and self-frequency shift in the HNL-DSF, which will speed the SC growth. ${ }^{10}$ New DWs are also expected to be generated as the solitons adjust their characteristics to the second fiber span. In fact, the frequency shift $\delta \omega$ at which DWs are generated, $\delta \omega \simeq-3 \beta_{2} / \beta_{3} \simeq 3\left(\omega_{\mathrm{ZDW}}-\omega\right)$, increases with the separation between the soliton carrier frequency $\omega$ and the $\mathrm{ZDW}^{3,8}\left(\beta_{3}\right.$ is the third-order dispersion coefficient). Since the solitons have already been shifted away from the ZDW in the first fiber span, the corresponding DWs will be generated further toward the blue and extend the SC toward short wavelengths. Note that we could in principle achieve a similar increase in $\delta \omega$ by using a single fiber with a larger $\beta_{2}$ coefficient, but this would reduce the initial spectral broadening due to MI. Using dual-wavelength pumping (1455 and $1555 \mathrm{~nm}$ ) in the two-fiber arrangement gives rise to an even broader SC that now spans from $1.4 \mu \mathrm{m}$ to more than $1.7 \mu \mathrm{m}$ (measured at $-20 \mathrm{~dB}$ ), as shown by the experimental data plotted at the top of Fig. 3. The SC spectrum is nearly flat above the $1555 \mathrm{~nm}$ RFL1 pump wavelength. However, the generated spectral density decreases quasi-exponentially toward the short wavelengths, in agreement with the 
theory predicting a lower efficiency for shortwavelength DWs. ${ }^{3,8}$

To spread the SC further, especially on the shortwavelength side, we have used our four fibers and three pump wavelengths together. The first previously filtered Raman order of RFL1 at $1289 \mathrm{~nm}$ with a peak power of $\sim 1 \mathrm{~W}$ amplifies through SRS the part of the SC at $\sim 1.3 \mu \mathrm{m}$. Simultaneously, since this pump wavelength is close to the ZDW of the SMF, new DWs are generated at even shorter wavelengths in the span of the SMF. The resulting SC can be observed in Fig. 4, which illustrates the SC spectral dynamics at the output of the four-fiber arrangement for an increasing total pump power. Note that the RFL1 power is increased first before switching on the $1455 \mathrm{~nm}$ RFL2. As can be seen, the $1289 \mathrm{~nm}$ pump line is separated from the main SC at low power but broadens and merges with it when launched together with RFL2, leading to an ultrabroadband SC at full power. To measure the full SC spectrum beyond the upper limit of our OSA, we set up another spectrometer made up of a transmission diffraction grating (300 grooves $/ \mathrm{mm}, 1 \mathrm{~nm}$ resolution) coupled to an extended long-wavelength InGaAs photodiode. The result is plotted in Fig. 5 on logarithmic and linear (inset) scales. This figure reveals that our combined technique leads to the generation of a $2 \mathrm{~W}$ ultrabroadband cw-SC with a $800 \mathrm{~nm}$ spectral extent, from 1.2 to $2.0 \mu \mathrm{m}$ (measured at $-5 \mathrm{~dB}$ ). Note in particular the excellent flatness of the SC spectrum above $1.6 \mu \mathrm{m}$, a behavior that has been already observed in the experiment of Ref. 4. We must also point out that we have tested the three-pump combination in all our fiber arrangements, and the most noticeable improvement in the SC was obtained for the configuration discussed here.

To conclude, we have experimentally shown that combining a multiwavelength pumping scheme and dispersion management can result in the stable generation of an ultrabroadband ( $800 \mathrm{~nm}$ wide) multiwatt $\mathrm{cw}$ supercontinuum that could directly find practical interests for numerous applications. The spectral broadening is achieved through Ramanassisted MI, soliton compression and DW generation. Our results suggest that using a multiorder Raman fiber laser, which can actually also be obtained through dispersion management of Raman laser

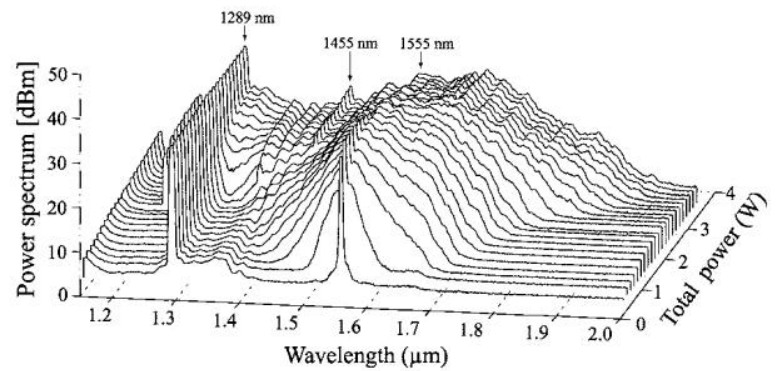

Fig. 4. Experimental output spectra for increasing total pump power showing the SC generation with multiwavelength pumping and dispersion management in the fourfiber arrangement. RFL2 is switched on after RFL1 reaches its maximum power. The OSA's wavelength limit has been extended to $2 \mu \mathrm{m}$.

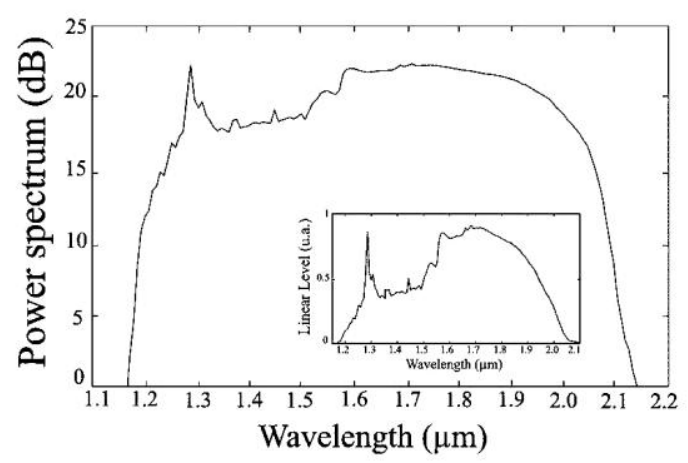

Fig. 5. SC spectrum in $\mathrm{dB}$ units measured with the extended InGaAs photodiode at maximum launch power. Inset, output spectrum in linear scale.

cavities, ${ }^{15}$ with a well-suited nonlinear fiber arrangement has tremendous potential applications. Using microstructured fibers instead of step-index fibers could also provide more freedom to manage dispersion or to make our technique applicable to shorter infrared and visible pump wavelengths.

This work has been supported by the French Ministry of Research and Technologie under contract ACI-JC801 and the Marsden Fund of the Royal Society of New Zealand. The authors thank A. Garnache for providing the photodiode. T. Sylvestre's e-mail address is thibaut.sylvestre@univ-fcomte.fr.

\section{References}

1. M. Prabhu, A. Taniguchi, S. Hirose, J. Lu, M. Musha, A. Shirakawa, and K. Ueda, Appl. Phys. B 77, 205 (2003).

2. M. González-Herráez, S. Martín-López, P. Corredera, M. L. Hernanz, and P. R. Horche, Opt. Commun. 226, 323 (2003)

3. A. Mussot, E. Lantz, H. Maillotte, T. Sylvestre, C. Finot, and S. Pitois, Opt. Express 12, 2838 (2004).

4. P. A. Champert, V. Couderc, and A. Barthélémy, IEEE Photon. Technol. Lett. 16, 2445 (2004).

5. A. K. Abeeluck and C. Headley, Opt. Lett. 30, 61 (2005).

6. F. Vanholsbeeck, S. Martín-Lopez, M. GonzálezHerráez, and S. Coen, Opt. Express 13, 6615 (2005).

7. S. V. Popov, J. R. Taylor, A. B. Rulkov, and V. P. Gapontsev, in Conference on Lasers and Electro-Optics (CLEO), Vol. 96 of OSA Trends in Optics and Photonics Series (Optical Society of America, 2004), paper CThEE4.

8. E. A. Golovchenko, P. V. Mamyshev, A. N. Pilipetskii, and E. M. Dianov, J. Opt. Soc. Am. B 8, 1626 (1991).

9. N. Akhmediev and M. Karlsson, Phys. Rev. A 51, 2602 (1995).

10. A. K. Abeeluck and C. Headley, Appl. Phys. Lett. 85, 4863 (2004).

11. J. N. Kutz, C. Lyngå, and B. J. Eggleton, Opt. Express 13, 3989 (2005).

12. J. C. Travers, S. V. Popov, and J. R. Taylor, Opt. Lett. 30, 3132 (2005).

13. T. Sylvestre, H. Maillotte, E. Lantz, and P. Tchofo Dinda, Opt. Lett. 24, 1561 (1999).

14. F. Vanholsbeeck, S. Coen, Ph. Emplit, C. Martinelli, and T. Sylvestre, Opt. Lett. 29, 998 (2004).

15. F. Vanholsbeeck, S. Coen, Ph. Emplit, C. Martinelli, F. Leplingard, and T. Sylvestre, Opt. Lett. 29, 2719 (2004). 